\title{
Comparative analysis of first-line treatment regimens for advanced EGFR-mutant non-small cell lung cancer patients with stable brain metastases
}

\author{
Lu Dai ${ }^{1 \#} \wedge$, Chun-Yue Luo ${ }^{2 \#}$, Guang-Xia Hu ${ }^{3}$, Gang Chen ${ }^{4}$, Chuan-Xin Wu ${ }^{1}$, Jun Yin ${ }^{1}$, Ze-Yong Jiang ${ }^{1}$, \\ Guang-Fu $\mathrm{Hu}^{5}$, Jian Zhao ${ }^{1}$, Wen-Fan Fu ${ }^{1}$
}

${ }^{1}$ Department of Thoracic Surgery, ${ }^{2}$ Department of Ultrasound Imaging, Affiliated Cancer Hospital \& Institute of Guangzhou Medical University, Guangzhou, China; ${ }^{3}$ Department of Pathology, Binzhong People's Hospital, Affiliated to First Shandong Medical University, Binzhong, China; ${ }^{4}$ Department of Thoracic Surgery, Guangdong General Hospital, Guangdong Academy of Medical Sciences, Guangzhou, China; ${ }^{5}$ Department of Breast Surgery, Huangpu Branch, Shanghai Ninth People’s Hospital, Affiliated to Shanghai Jiao Tong University School of Medicine, Shanghai, China

Contributions: (I) Conception and design: L Dai; (II) Administrative support: J Zhao; (III) Provision of study materials or patients: WF Fu; (IV) Collection and assembly of data: GX Hu; (V) Data analysis and interpretation: CY Luo; (VI) Manuscript writing: All authors; (VII) Final approval of manuscript: All authors.

\#These authors contributed equally to this work.

Correspondence to: Wen-Fan Fu. Department of Thoracic Surgery, Affiliated Cancer Hospital \& Institute of Guangzhou Medical University, Guangzhou 510095, China. Email: tgzy2019@outlook.com; Jian Zhao. Department of Thoracic Surgery, Affiliated Cancer Hospital \& Institute of Guangzhou Medical University, Guangzhou 510095, China. Email: zj_hjh@163.com.

Background: To compare the survival outcomes of first-line treatment regimens for advanced epidermal growth factor receptor (EGFR)-mutant non-small cell lung cancer (NSCLC) patients with stable brain metastases.

Methods: We conducted a systematic review of available data from randomized controlled trials (RCTs) of first-line treatment regimens of NSCLC patients with stable brain metastases. Progression free survival (PFS) and overall survival (OS) were extracted and analysed from the RCT subgroups. A network meta-analysis was constructed using the Bayesian statistical model to synthesize the survival outcomes of all the treatments.

Results: The analysis included 6 eligible RCT subgroups with 417 patients and 7 treatment regimens osimertinib, afatinib, first-generation EGFR-TKI (gefitinib or erlotinib), erlotinib + bevacizumab, gefitinib + pemetrexed + carboplatin, gemcitabine + cisplatin, and pemetrexed + cisplatin. Of these seven treatment regimens, gefitinib + pemetrexed + carboplatin had the highest potential for favorable PFS and OS, followed by osimertinib, in the treatment of advanced EGFR-mutant NSCLC patients with stable brain metastases. None of the results met the predetermined statistical significance of $\mathrm{P}<0.05$.

Conclusions: The regimens of "Gefitinib + pemetrexed + carboplatin" and "Osimertinib" were associated with the most favorable PFS and OS compared to the other therapies in advanced EGFR-mutant NSCLC patients with stable brain metastases, although the difference between these regimens and the others was not statistically significantly different.

Keywords: Epidermal growth factor receptor (EGFR); brain metastases; combination treatments; network metaanalysis; progression free survival; overall survival (OS)

Submitted Apr 21, 2020. Accepted for publication Jul 04, 2020.

doi: 10.21037/apm-20-1136

View this article at: http://dx.doi.org/10.21037/apm-20-1136

$\wedge$ ORCID: 0000-0002-6895-9619 


\section{Introduction}

Lung cancer has the highest incidence and mortality rate of any cancer worldwide (1). Non-small cell lung cancer (NSCLC) comprises approximately $85 \%$ of all lung cancer cases (2). Epidermal growth factor receptor (EGFR) gene mutations are carried by around $50 \%$ of NSCLC patients in East Asian countries (3) and about $10-15 \%$ of the Caucasian NSCLC patients (4). EGFR mutations are more commonly found in women, East Asians, adenocarcinoma patients, and people who have never smoked (5). Interestingly, for advanced EGFR-mutant NSCLC patients, EGFR tyrosine kinase inhibitor (TKIs) therapy, rather than chemotherapy, is preferred (6). There are a number of different generations of EGFR-TKIs. First-generation EGFR-TKIs include erlotinib, gefitinib, and icotinib (which was made in China) (7), while dacomitinib and afatinib are second-generation EGFR-TKIs. Meanwhile, third-generation EGFR-TKIs include osimertinib, which has been used as a first-line antiNSCLC therapy. Furthermore, EGFR-TKIs have been used in combination with other treatments (chemotherapy, monoclonal antibodies, and immunotherapy) as first-line treatment to improve efficacy and curb drug resistance (8).

Brain metastasis is one of the leading causes of lung cancer mortality (9). Of NSCLC patients, 20\% have brain metastases at diagnosis, and approximately $25-50 \%$ of patients develop metastasis during the course of their disease (10). The blood-brain barrier protects the central nervous system and restricts drug access, which makes NSCLC patients with brain metastases a special group (11). The main treatments for NSCLC with brain metastases are local therapy (WBRT (whole brain radiation therapy), SRS (stereotactic radiotherapy), surgery), and systemic therapy (chemotherapy, gene target therapy, immunotherapy and combination therapy). For symptomatic/uncontrolled and stable brain metastases, local and systemic therapy are the recommended first-line treatments, respectively $(7,12)$. Stable brain metastases refers to clinically asymptomatic and controlled brain metastases [defined in (randomized controlled trials) RCTs as metastases that are asymptomatic and/or do not require treatment with anticonvulsants or steroids and/or no leptomeningeal disease, and/or symptomatic/uncontrolled metastases that remained stable for at least four weeks after local brain radiotherapy or surgery prior to randomization]. For EGFR-mutant NSCLC patients with stable brain metastases, first-line options include osimertinib, gefitinib, erlotinib, afatinib, or a combination regimen (erlotinib + bevacizumab or gefitinib + pemetrexed + carboplatin). Previous study has performed preclinical comparison of osimertinib with other EGFR-TKIs in EGFR-mutant NSCLC brain metastases models, and early evidence of clinical brain metastases activity (13); previous network meta-analyse has compared all first-line treatments for patients with advanced EGFRmutant NSCLC (14); however, data comparing the survival outcomes of the current first-line treatments in EGFRmutant NSCLC patients with stable brain metastases is limited.

We conducted a network meta-analysis of RCTs to provide insight into the relative survival outcomes of firstline treatments for EGFR-mutant NSCLC patients with stable brain metastases. We present the following article in accordance with the PRISMA reporting checklist (available at http://dx.doi.org/10.21037/apm-20-1136).

\section{Methods}

\section{Data sources and search strategy}

We conducted our study based on the Preferred Reporting Items for Systematic Reviews and Meta-Analyses (PRISMA) extension (15). In brief, we performed a systematic search of the PubMed, EMBASE, Cochrane Library, and ClinicalTrials.gov databases for relevant articles up to 25 November, 2019. There were no language restrictions. In addition, supplementary searches of conference proceedings of the American Society of Clinical Oncology, European Society of Medical Oncology, European Cancer Conference, and World Conference on Lung Cancer from between 2014 and 2019 were also carried out. The search took into account both Medical Subject Headings (MeSH) and text words, using the main search terms "NSCLC" and "EGFR", and was limited to "RCT". The full search strategy is provided in the Table $S 1$.

\section{Study selection}

The titles and abstracts of all the studies that were initially returned were screened, and the full papers of studies were assessed for final selection.

We included phase II/III randomised controlled trials that met the following criteria: (I) studies that enrolled patients with histologically or cytologically confirmed advanced (stage III/IV/recurrent) NSCLC harboring activating EGFR mutations, including Leu858Arg, exon 19 deletions, and others, were included for analysis. (II) 
We also included studies that compared two or more first-line treatments in patients with advanced EGFRmutant NSCLC. (III) Studies that enrolled and performed subgroup analysis of patients with brain metastases. (IV) Studies that reported either progression-free survival (PFS) or overall survival (OS).

We excluded studies not adhering to the inclusion criteria. Other exclusion criteria were: (I) studies that enrolled patients with symptomatic/uncontrolled brain metastases were excluded. (II) Patients who received treatments other than those approved by any food and drug administration were excluded. (III) Studies that did not report hazard ratio (HR) were also excluded.

\section{Data extraction and quality assessment}

We evaluated RCTs involving advanced EGFR-mutant NSCLC patients with stable brain metastases and the treatment recommendations in the China expert consensus (12), which were based on expert interpretations of the RCT subgroup data. The data of the brain metastases subgroup from the RCTs of patients with advanced EGFR-mutant NSCLC were extracted for statistical analysis and systematic review. For unreported data, we contacted the authors or the pharmaceutical companies.

The quality and risk of bias of the individual RCTs were assessed using the Cochrane Collaboration's riskof-bias tool (16). The tool is based on random sequence generation, allocation concealment, blinding of participants and personnel, blinding of outcome assessment, incomplete outcome data, selective outcome reporting, and other sources of bias. The RCTs were judged as having low, high, or unclear risk of bias.

Study selection and data extraction were managed by all authors independently. Two authors (LD and CL) independently assessed risk of bias in the individual studies. Any disagreement between the two authors were resolved by consensus and arbitration by a panel of adjudicators (LD, CL, GH, GC, CW, JY, ZJ, GH, JZ and WF).

\section{Statistical analyses}

The risk of bias in the RCTs was assessed by Review Manager (RevMan, 5.3, The cochrane collaboration, London, UK). The data on HRs with $95 \%$ credible interval were extracted from the included studies. The network plots were drawn using GeMTC package in R software (version 3.6.3, R Foundation, Vienna, Austria) to show the interaction of the different treatment regimens in the included studies. The direct and indirect treatments in our network meta-analysis and their effects were analyzed using the Bayesian statistical model. Statistical significance was set at a $\mathrm{P}=0.05$. Statistical heterogeneity was estimated by means of the $\mathrm{I}^{2}$ statistic, which indicates heterogeneity caused by total variation across trials rather than by chance. Values $<25 \%$ indicate a small amount of inconsistency while values $>50 \%$ indicate a huge amount of inconsistency (17).

\section{Results}

\section{Study selection and study quality}

We electronically and manually searched a total of 2,025 records. After the titles and abstracts were screened, 1,793 records were excluded. Of the 151 abstracts and manuscripts that qualified for full-text reading, only six unique RCTs finally included for analysis are shown in Figure 1.

According to the Cochrane Collaboration's risk-of-bias tool, all six RCTs in our study were considered to be of acceptable quality and have low risk of bias. The main risk was that all the data came from subgroups. Figure $S 1$ and Table $S 2$ show the risk of bias assessments in detail.

\section{Systematic review and characteristics}

None of the RCTs specifically focused on advanced EGFRmutant NSCLC patients with stable brain metastases. We therefore collected data from subgroups of patients with advanced EGFR-mutant NSCLC. The six RCTs included were: (FLAURA (18,19), NEJ026 (20), NEJ009 (21), LUXlung7 (22,23), LUX-lung6 (24), and LUX-lung3 (24). These studies involved 417 patients, who received 7 different first-line regimens: osimertinib, first-generation EGFR-TKI (We defined "erlotinib or gefitinib" as firstgeneration EGFR-TKI), afatinib, gemcitabine + cisplatin, pemetrexed + cisplatin, erlotinib + bevacizumab and gefitinib + pemetrexed + carboplatin. The overall study and the brain metastases subgroup characteristics are shown in Table 1 and Table 2, respectively.

In the FLAURA study, patients with central nervous system metastases whose condition was neurologically stable were eligible; most of these patients had brain metastases, but we could not exclude the small number of patients without brain metastases. 


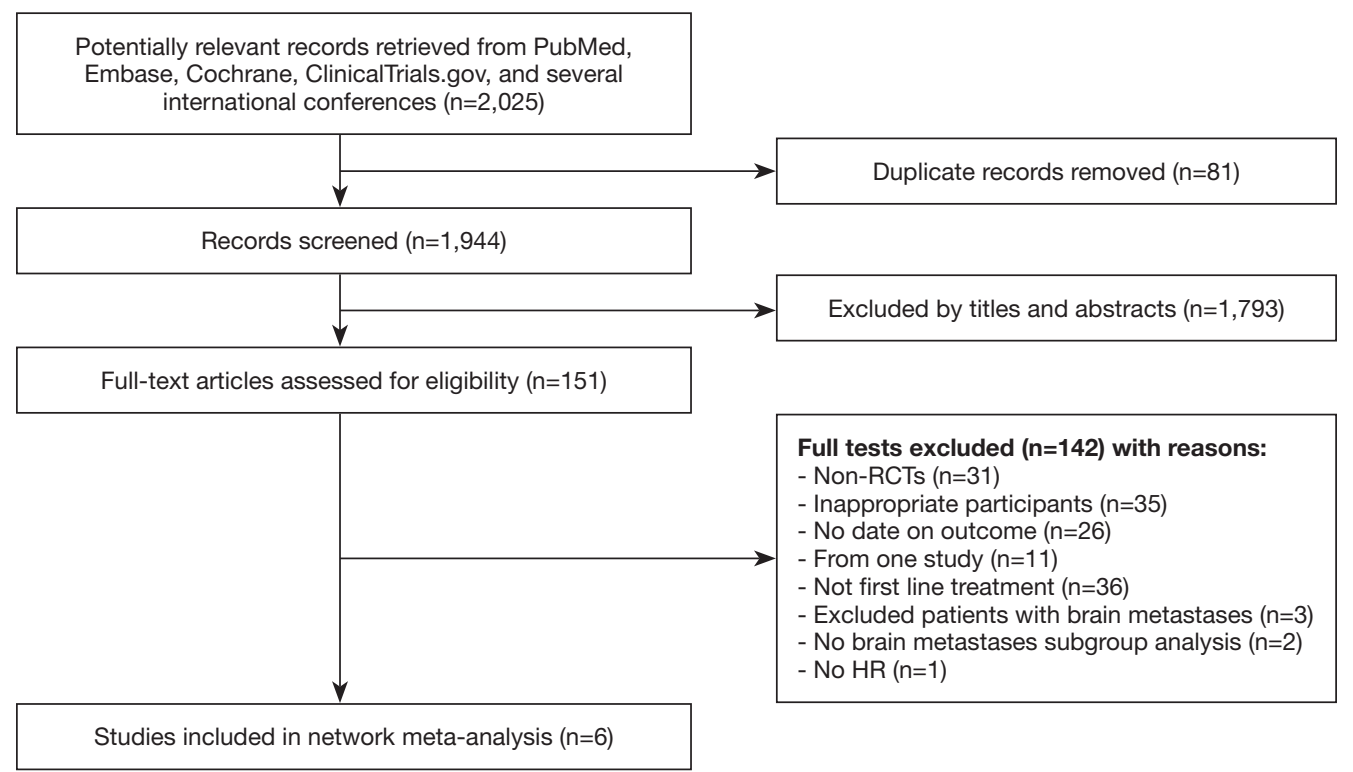

Figure 1 Flow chart of the study selection. RCT, randomized controlled trial; HR, hazard ratio.

Table 1 Overall characteristics of the included studies

\begin{tabular}{|c|c|c|c|c|c|c|c|c|}
\hline $\begin{array}{l}\text { Study } \\
\text { (phase) }\end{array}$ & Ethnicity & $\begin{array}{l}\text { Sample } \\
\text { size }\end{array}$ & $\begin{array}{c}\text { Female } \\
(\%)\end{array}$ & $\begin{array}{l}\text { Median } \\
\text { age }\end{array}$ & EGFR mutation types & Intervention arm & Control arm & $\begin{array}{l}\text { Reported } \\
\text { outcomes }\end{array}$ \\
\hline $\begin{array}{l}\text { FLAURA, } \\
2019 \text { (III) }\end{array}$ & Multiple & $279 / 277$ & $64.0 / 62.0$ & $64.0 / 64.0$ & 19 deletion, L858R & Osimertinib & $\begin{array}{l}\text { Gefitinib or } \\
\text { Erlotinib }\end{array}$ & $\begin{array}{c}\text { PFS; OS; ORR; } \\
\text { Grade } \geq 3 \text { Aes }\end{array}$ \\
\hline $\begin{array}{l}\text { NEJ026, } \\
2019 \text { (III) }\end{array}$ & Asian & $114 / 114$ & $63.4 / 65.2$ & $67.0 / 68.0$ & 19 deletion, L858R & $\begin{array}{c}\text { Erlotinib + } \\
\text { bevacizumab }\end{array}$ & Erlotinib & $\begin{array}{c}\text { PFS; ORR; } \\
\text { Grade } \geq 3 \text { Aes }\end{array}$ \\
\hline $\begin{array}{l}\text { LUX-lung7, } \\
2017 \text { (IIB) }\end{array}$ & Asian & $160 / 159$ & $57.0 / 67.6$ & 63.0/63.0 & 19 deletion, L858R & Afatinib & Gefitinib & $\begin{array}{c}\text { PFS; OS; ORR; } \\
\text { Grade } \geq 3 \text { Aes }\end{array}$ \\
\hline $\begin{array}{l}\text { LUX-lung6, } \\
2016 \text { (III) }\end{array}$ & Asian & $242 / 122$ & $64.0 / 68.0$ & $58.0 / 58.0$ & $\begin{array}{l}19 \text { deletion, L858R and } \\
\text { other mutations }\end{array}$ & Afatinib & $\begin{array}{l}\text { Gemcitabine + } \\
\text { cisplatin }\end{array}$ & $\begin{array}{c}\text { PFS; OS; ORR; } \\
\text { Grade } \geq 3 \text { Aes }\end{array}$ \\
\hline
\end{tabular}

Data are expressed as intervention/control unless indicated otherwise. PFS, progression free survival; OS, overall survival; ORR, objective response rate; Aes, adverse events; PC, pemetrexed + carboplatin; NR, not reported; HR, hazard ratio.

\section{Network meta-analysis}

We constructed a complete network meta-analysis that included 6 RCTs, comprising of 7 treatments: osimertinib, first-generation EGFR-TKI, Erlotinib + bevacizumab, gefitinib + pemetrexed + carboplatin, afatinib, gemcitabine + cisplatin, or pemetrexed + cisplatin for PFS, and 6 treatments: osimertinib, first-generation EGFR-TKI, gefitinib + pemetrexed + carboplatin, afatinib, gemcitabine
+ cisplatin, or pemetrexed + cisplatin for OS (Figure 2).

The combination of gefitinib + pemetrexed + carboplatin yielded a better PFS rate than osimertinib (HR: 0.84; 95\% CI: 0.34 to 2.11 ), first-generation EGFR-TKIs (erlotinib or gefitinib) (HR: $0.61 ; 95 \%$ CI: 0.32 to 1.17 ), erlotinib + bevacizumab (HR: 0.68 ; $95 \%$ CI: 0.28 to 1.67 ), afatinib (HR: 0.69; $95 \%$ CI: 0.28 to 1.71 ), gemcitabine + cisplatin (HR: 0.50, $95 \%$ CI: 0.15 to 1.67$)$, and pemetrexed + cisplatin 
Table 2 Brain metastases subgroup characteristics of the included studies

\begin{tabular}{lccccc}
\hline Study & Sample size & Intervention arm & Control arm & PFS (HR; 95\% Cl) & OS (HR; 95\% Cl) \\
\hline FLAURA, 2019 & $53 / 63$ & Osimertinib & Gefitinib or Erlotinib & $0.47(0.30-0.74)$ & $0.830 .53-1.30$ \\
NEJ026, 2019 & $36 / 36$ & Erlotinib + bevacizumab & Erlotinib & $0.78(0.42-1.43)$ \\
NEJ009, 2019 & $38 / 50$ & Gefitinib + PC & Gefitinib & $0.32(0.19-0.53)$ & $0.66(0.40-1.07)$ \\
LUX-lung7, 2017 & $26 / 24$ & Afatinib & Gefitinib & $0.76(0.41-1.44)$ & $1.16(0.61-2.21)$ \\
LUX-lung6, 2016 & $30 / 19$ & Afatinib & Gemcitabine + cisplatin & $0.47(0.18-1.21)$ & $1.13(0.56-2.26)$ \\
LUX-lung3, 2016 & $27 / 15$ & Afatinib & Pemetrexed + cisplatin & $0.54(0.23-1.25)$ & $1.15(0.49-2.67)$ \\
\hline
\end{tabular}

PFS, progression free survival; HR, hazard ratio.
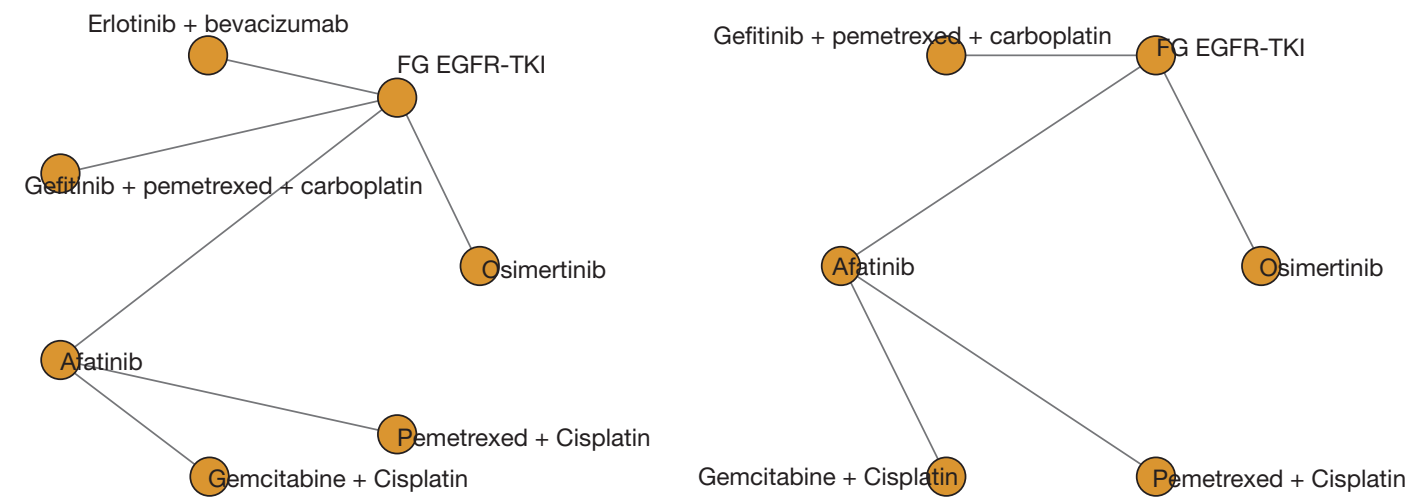

Figure 2 Complete network based on six RCTs. (A) Comparisons of progression-free survival in advanced EGFR-mutant NSCLC patients with stable brain metastases. (B) Comparisons of overall survival in advanced EGFR-mutant NSCLC patients with stable brain metastases. EGFR epidermal growth factor receptor; RCT, randomized controlled trial; NSCLC, non-small cell lung cancer.

(HR: 0.52 ; 95\% CI: 0.17 to 1.66) (Figure 3). Compared to the other EGFR-TKI monotherapies, osimertinib yielded the greatest benefit. Furthermore, gefitinib + pemetrexed + carboplatin and osimertinib had the first (57\%) and second (37\%) highest performance probability, respectively (Figure 4 and Table S3). Two chemotherapy regimens (gemcitabine + cisplatin and pemetrexed + cisplatin) had the worst PFS rate. Notably, none of the results achieved the predetermined statistical significance of $\mathrm{P}<0.05$.

Our data showed that gefitinib + pemetrexed + carboplatin yielded a better OS rate than osimertinib (HR: 0.91; $95 \%$ CI: 0.60 to 1.37), first-generation EGFR-TKIs (erlotinib or gefitinib) (HR: 0.84; 95\% CI: 0.62 to 1.13 ), afatinib (HR: 0.78 ; $95 \%$ CI: 0.49 to 1.25 ), gemcitabine + cisplatin (HR: $0.82 ; 95 \%$ CI: 0.46 to 1.49 ), and pemetrexed + cisplatin (HR: 0.84; 95\% CI: 0.45 to 1.56 ) (Figure 3). Gefitinib + pemetrexed + carboplatin had a $47 \%$ chance of being the best; Compared to the other EGFR-TKIs monotherapies, osimertinib yielded the greatest benefit, with a performance probability of $29 \%$ (Figure 5 and Table S3). As with PFS rate, gemcitabine + cisplatin and pemetrexed + cisplatin had the worst OS rate. Notably, none of the results achieved the predetermined statistical significance of $\mathrm{P}<0.05$.

Further analysis showed low global heterogeneity for both the PFS $\left(I^{2}=16 \%\right)$ and OS $\left(I^{2}=18 \%\right)$ rates.

\section{Discussion}

In this study, we investigated the relative survival outcomes (PFS and OS rates) of each of the first-line regimens currently used to treat advanced EGFR-mutant NSCLC patients with stable brain metastases. The results suggested that gefitinib + pemetrexed + carboplatin provided the best PFS and OS rates for such patients. Compared to the other EGFR-TKIs monotherapies, Osimertinib achieved the best PFS and OS rates in patients with advanced EGFRmutant NSCLC with stable brain metastases. Meanwhile, 


\begin{tabular}{lllllll}
\hline PFS & & & & & & \\
Osimertinib & $0.72(0.38,1.35)$ & $0.81(0.33,1.97)$ & $1.19(0.47,2.92)$ & $0.82(0.33,2.03)$ & $0.59(0.16,1.97)$ & $0.62(0.20,1.94)$ \\
$1.38(0.74,2.60)$ & FG EGFR-TKI & $1.11(0.58,2.13)$ & $1.64(0.86,3.10)$ & $1.13(0.59,2.18)$ & $0.82(0.29,2.28)$ & $0.86(0.34,2.22)$ \\
$1.24(0.51,3.06)$ & $0.90(0.47,1.72)$ & Erlotinib + bevacizumab & $1.48(0.60,3.60)$ & $1.02(0.40,2.56)$ & $0.74(0.22,2.45)$ & $0.77(0.24,2.45)$ \\
$0.84(0.34,2.11)$ & $0.01(0.32,1.17)$ & $0.08(0.28,1.67)$ & Gefitinib+ PC & $0.69(0.28,1.71)$ & $0.50(0.15,1.67)$ & $0.52(0.17,1.60)$ \\
$1.22(0.49,3.07)$ & $0.89(0.46,1.70)$ & $0.98(0.39,2.51)$ & $1.45(0.58,3.63)$ & Afatinib & $0.73(0.33 .1 .59)$ & $0.76(0.38,1.52)$ \\
$1.69(0.51,5.69)$ & $1.22(0.44,3.42)$ & $1.35(0.41,4.61)$ & $2.00(0.60,6.72)$ & $1.38(0.63,3.03)$ & Gemcitabine + cis & $1.06(0.37,3.03)$ \\
$1.61(0.51,4.99)$ & $1.16(0.45,296)$ & $1.29(0.41,4.09)$ & $1.91(0.60,5.95)$ & $1.31(0.66,2.61)$ & $0.94(0.33,2.71)$ & Pemetrexed + cis \\
\hline OS & & & & & & \\
Osimertinib & $0.92(0.69,1.23)$ & - & $1.1(0.73,1.68)$ & $0.87(0.55,1.37)$ & $0.91(0.51,1.66)$ & $0.93(0.50,1.73)$ \\
$1.08(0.81,1.44)$ & FG EGFR-TKI & - & $1.19(0.89,1.61)$ & $0.94(0.66,1.33)$ & $0.98(0.59,1.64)$ & $1.01(0.58,1.75)$ \\
$0.91(0.60,1.37)$ & $0.84(0.62,1.13)$ & - & Gefitinib+ PC & $0.78(0.49,1.25)$ & $0.82(0.46 .1 .49)$ & $0.84(0.45,1.50)$ \\
$1.16(0.73,1.83)$ & $1.07(0.75,1.52)$ & - & $1.28(0.80,2.03)$ & Afatimib & $1.05(0.73,1.52)$ & $1.07(0.71,1.02)$ \\
$1.10(0.60,1.97)$ & $1.02(0.61,1.68)$ & - & $1.21(0.67,2.19)$ & $0.95(0.66,1.37)$ & Gemcitabiunc + cis & $1.02(0.59,1.76)$ \\
$1.08(0.58,2.01)$ & $0.99(0.57,1.72)$ & - & $1.19(0.64,2.23)$ & $0.93(0.62,1.41)$ & $0.98(0.57,1.69)$ & Pemetrexed + cis
\end{tabular}

Figure 3 Treatment comparisons for PFS, OS [HRs (95\% CI)]. PFS, progression free survival; OS, overall survival; NG, not given; HR, hazard ratio; FG, first-generation; PC, pemetrexed + carboplatin; EGFR epidermal growth factor receptor; cis, cisplatin.

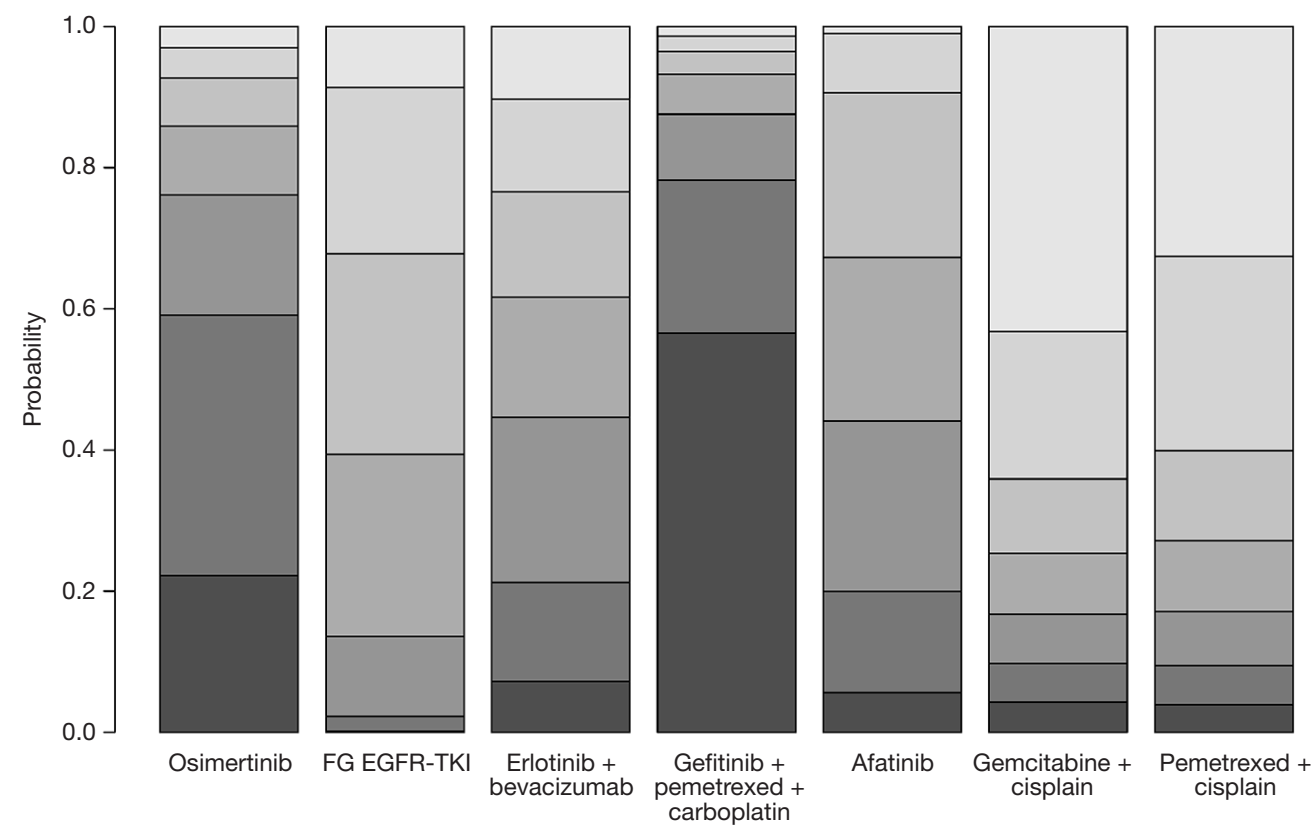

Figure 4 Bayesian ranking profiles of treatments based on PFS. FG, first-generation; PFS, progression free survival.

the chemotherapy regimens (gemcitabine + cisplatin and pemetrexed + cisplatin) had the poorest PFS and OS rates.

Previous network meta-analyses (14) that compared all first-line treatments for advanced EGFR-mutant NSCLC patients found that gefitinib + pemetrexed-based chemotherapy and osimertinib were associated with the greatest benefits for PFS and OS. Gefitinib + pemetrexed based chemotherapy was also associated with the best objective response rate. The combinations of erlotinib + bevacizumab and gefitinib + pemetrexed were associated 

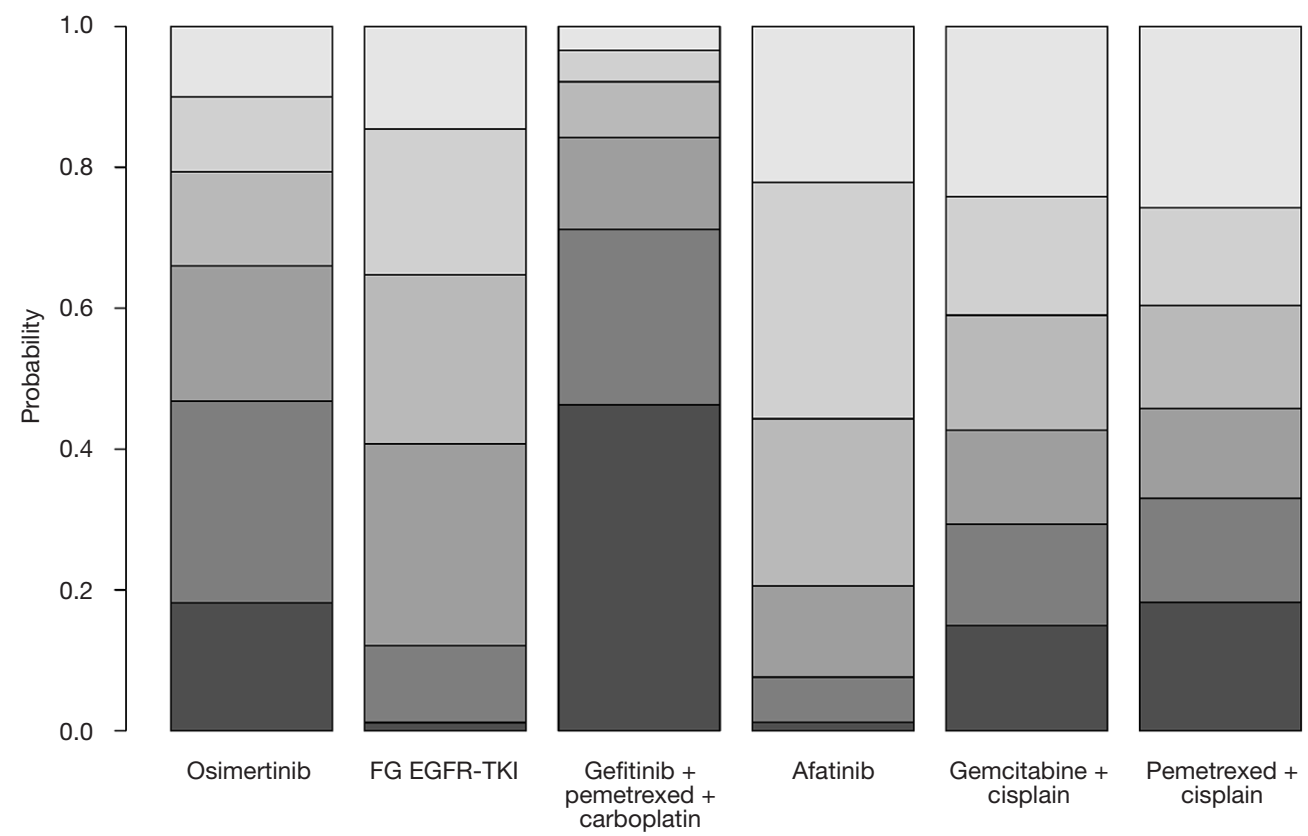

Figure 5 Bayesian ranking profiles of treatments based on OS. FG, first-generation; OS, overall survival.

with increased risk of grade 3 adverse events, compared to the other EGFR-TKI monotherapies. Data from our study suggested that a combination of gefitinib, pemetrexed, and carboplatin was the most favorable regimen in terms of PFS (with $57 \%$ probability of being the best; Figure 3 and Table S3) and OS (with $47 \%$ probability of being the best; Figure 4 and Table S3), although this was not statistically significant. In vitro and in vivo studies have demonstrated that a combination of EGFR-TKIs and chemotherapy produces a synergistic effect (25-29).

The FLAURA study showed that, compared with standard EGFR-TKIs, osimertinib improved both the median PFS (18.9 vs. 10.2 months) and the overall OS (38.6 vs. 31.8 months) for advanced EGFR-mutant NSCLC patients $(18,19)$. Based on the results of the FLAURA study, the NCCN clinical practice guidelines recommended osimertinib as a first-line therapy for patients with advanced EGFR-mutant NSCLC (7). In addition, a network metaanalysis showed that, compared to the other TKIs, osimertinib had a potentially better efficacy (in relation to both PFS and OS) as a first-line treatment for advanced EGFR-mutant NSCLC patients (30). Furthermore, subgroup analysis of CNS metastases reported that osimertinib had better efficacy than and reduced the risk of CNS progression compared to standard EGFR-TKI (31). Consequently, Shanghai expert consensus recommended osimertinib as a first-line treatment for advanced EGFRmutant NSCLC with stable brain metastases (12).

Our study performed network meta-analysis to compare all of the first-line treatments in subgroup data. Our data showed that while osimertinib was associated with better survival outcomes compared to the other EGFR-TKI monotherapies, a combination of gefitinib + pemetrexed + carboplatin was potentially better than osimertinib (PFS, HR: $0.84,95 \%$ CI: 0.34 to 2.11 ; OS, HR: $0.91,95 \%$ CI: 0.60 to 1.37 ), as shown in Figure 3. These results suggest that a combination therapy with osimertinib may be more effective than the current standard regimens. Preliminary data from ongoing studies have shown that a combination of osimertinib plus ramucirumab has therapeutic potential when used as a second-line treatment for EGFR-mutant, T790M-positive NSCLC patients with brain metastases (32). A phase $1 / 2$ study of osimertinib plus bevacizumab as a first-line treatment regimen for patients with EGFR-mutant NSCLC observed a PFS of 18.4 months (33). A retrospective analysis of osimertinib combined with chemotherapy showed an improvement in the CNS metastasis (34). However, osimertinib had a much improved toxicity profile compared to gefitinib plus pemetrexed-based chemotherapy. Compared to the gefitinib plus pemetrexed regimen, osimertinib had fewer adverse events of grade 3 or higher in patients with advanced EGFR mutated NSCLC (14). In addition, a recent meta- 
analysis involving 15 studies supported the potential role of osimertinib in the treatment of advanced EGFR-mutant NSCLC patients with intracranial metastases (35). Based on the current evidence, we put forward the use of osimertinib as a first-line treatment for advanced EGFR-mutant NSCLC patients with stable brain metastases.

Several studies (36-42) have shown that EGFR-TKIs are superior to chemotherapy as first-line treatment for patients with advanced EGFR-mutant NSCLC. In our analysis, the chemotherapy regimens (gemcitabine + cisplatin and pemetrexed + cisplatin) were associated with less favorable outcomes in advanced EGFR-mutant NSCLC patients with stable brain metastases.

By analyzing evidence from all RCTs, our review provides clinicians with a reference by which to consider the survival outcomes of the current treatment options. The use of gefitinib + pemetrexed + carboplatin was associated with the most favorable survival outcomes and, as osimertinib had a better toxicity profile than gefitinib and pemetrexed-based chemotherapy (14), gefitinib + pemetrexed + carboplatin and osimertinib were both considered as optimal treatments for advanced EGFR-mutant NSCLC patients with stable brain metastases. Future RCTs should be designed to focus on potentially more effective combinations, such as osimertinib + chemotherapy or bevacizumab.

However, our study has some limitations. First, it was based on a small sample size and was retrospective in nature, which made it difficult to compare local heterogeneity between any two identical samples. Second, all of the data in our study was collected from subgroups; thus, the risk of bias cannot be ruled out. Third, analysis of adverse events and objective response rate proved challenging as this data was not available for the subgroups. Fourth, the demographic data of patients with brain metastases would have been helpful for evaluating differences between treatment groups but were not available. Fifth, the FLAURA study enrolled patients with CNS metastases whose condition was neurologically stable. While most of these patients had brain metastases, it was not possible to exclude the few patients without brain metastases from this analysis. Finally, our network meta-analysis assumed that the patient populations from different trials were similar, and that any differences would not substantially influence the results.

\section{Conclusions}

In summary, a regimen of gefitinib + pemetrexed + carboplatin was associated with the best PFS and OS in advanced EGFR-mutant NSCLC patients with stable brain metastases, followed by osimertinib. Gefitinib + pemetrexed + carboplatin and osimertinib could be optimal first-line treatments for patients with advanced EGFR-mutant NSCLC with stable brain metastases. This review of the literature available for current treatment regimens may provide insights for the design of future clinical trials.

\section{Acknowledgments}

The authors would like to acknowledge all the members of Affiliated Cancer Hospital \& Institute of Guangzhou Medical University.

Funding: This study was supported by grants from the National Natural Science Foundation of China (No. 81572258).

\section{Footnote}

Reporting Checklist: The authors have completed the PRISMA reporting checklist. Available at http://dx.doi. org/10.21037/apm-20-1136

Conflicts of Interest: All authors have completed the ICMJE uniform disclosure form (available at http://dx.doi. org/10.21037/apm-20-1136). The authors have no conflicts of interest to declare.

Ethical Statement: The authors are accountable for all aspects of the work in ensuring that questions related to the accuracy or integrity of any part of the work are appropriately investigated and resolved.

Open Access Statement: This is an Open Access article distributed in accordance with the Creative Commons Attribution-NonCommercial-NoDerivs 4.0 International License (CC BY-NC-ND 4.0), which permits the noncommercial replication and distribution of the article with the strict proviso that no changes or edits are made and the original work is properly cited (including links to both the formal publication through the relevant DOI and the license). See: https://creativecommons.org/licenses/by-nc-nd/4.0/.

\section{References}

1. Bray F, Ferlay J, Soerjomataram I, et al. Global cancer statistics 2018: GLOBOCAN estimates of incidence and mortality worldwide for 36 cancers in 185 countries. CA 
Cancer J Clin 2018;68:394-424.

2. Travis WD, Brambilla E, Noguchi M, et al. International association for the study of lung cancer/american thoracic society/european respiratory society international multidisciplinary classification of lung adenocarcinoma. J Thorac Oncol 2011;6:244-85.

3. Karachaliou N, Fernandez-Bruno M, Bracht JW, et al. EGFR first- and second-generation TKIs-there is still place for them in EGFR-mutant NSCLC patients. Transl Cancer Res 2019;8:S23-47.

4. Barlesi F, Mazieres J, Merlio JP, et al. Routine molecular profiling of patients with advanced non-small-cell lung cancer: results of a 1-year nationwide programme of the French Cooperative Thoracic Intergroup (IFCT). Lancet 2016;387:1415-26.

5. Rosell R, Moran T, Queralt C, et al. Screening for epidermal growth factor receptor mutations in lung cancer. N Engl J Med 2009;361:958-67.

6. Greenhalgh J, Dwan K, Boland A, et al. Firstline treatment of advanced epidermal growth factor receptor (EGFR) mutation positive non-squamous nonsmall cell lung cancer. Cochrane Database Syst Rev 2016;(5):CD010383.

7. NCCN clinical practice guidelines in oncology for nonsmall cell lung cancer. Version 2. 2020. Available online: https://www.nccn.org/patients. Accessed 23 December 2019.

8. Wu SG, Shih JY. Management of acquired resistance to EGFR TKI-targeted therapy in advanced non-small cell lung cancer. Mol Cancer 2018;17:38.

9. Nishino M, Soejima K, Mitsudomi T. Brain metastases in oncogene-driven non-small cell lung cancer. Transl Lung Cancer Res 2019;8:S298-307.

10. Ernani V, Stinchcombe TE. Management of Brain Metastases in Non-Small-Cell Lung Cancer. J Oncol Pract 2019;15:563-70.

11. Zhang Z, Guo H, Lu Y, et al. Anaplastic lymphoma kinase inhibitors in non-small cell lung cancer patients with brain metastases: a meta-analysis. J Thorac Dis 2019;11:1397-409.

12. Liang Xiaohua HR, Qiong Z. Shanghai expert consensus on the management of brain metastasis of non-small cell lung cancer with driver gene mutations. China Oncology 2019;29:71-80.

13. Ballard P, Yates JW, Yang Z, et al. Preclinical Comparison of Osimertinib with Other EGFR-TKIs in EGFR-Mutant NSCLC Brain Metastases Models, and Early Evidence of Clinical Brain Metastases Activity. Clin Cancer Res
2016;22:5130-40.

14. Zhao Y, Liu J, Cai X, et al. Efficacy and safety of first line treatments for patients with advanced epidermal growth factor receptor mutated, non-small cell lung cancer: systematic review and network meta-analysis. BMJ 2019;367:15460.

15. Hutton B, Salanti G, Caldwell DM, et al. The PRISMA extension statement for reporting of systematic reviews incorporating network meta-analyses of health care interventions: checklist and explanations. Ann Intern Med 2015;162:777-84.

16. Higgins JP, Altman DG, Gøtzsche PC, et al. The Cochrane Collaboration's tool for assessing risk of bias in randomised trials. BMJ 2011;343:d5928.

17. Higgins JP, Thompson SG, Deeks JJ, Altman DG. Measuring inconsistency in meta-analyses. BMJ 2003;327:557-60.

18. Soria JC, Ohe Y, Vansteenkiste J, et al. Osimertinib in Untreated EGFR-Mutated Advanced Non-Small-Cell Lung Cancer. N Engl J Med 2018;378:113-25.

19. Ramalingam SS, Vansteenkiste J, Planchard D, et al. Overall Survival with Osimertinib in Untreated, EGFR-Mutated Advanced NSCLC. N Engl J Med 2020;382:41-50.

20. Saito H, Fukuhara T, Furuya N, et al. Erlotinib plus bevacizumab versus erlotinib alone in patients with EGFR-positive advanced non-squamous non-small-cell lung cancer (NEJ026): interim analysis of an open-label, randomised, multicentre, phase 3 trial. Lancet Oncol 2019;20:625-35.

21. Hosomi Y, Morita S, Sugawara S, et al. Gefitinib Alone Versus Gefitinib Plus Chemotherapy for Non-Small-Cell Lung Cancer With Mutated Epidermal Growth Factor Receptor: NEJ009 Study. J Clin Oncol 2020;38:115-23.

22. Park K, Tan EH, O'Byrne K, et al. Afatinib versus gefitinib as first-line treatment of patients with EGFR mutationpositive non-small-cell lung cancer (LUX-Lung 7): a phase 2B, open-label, randomised controlled trial. Lancet Oncol 2016;17:577-89.

23. Paz-Ares L, Tan EH, O'Byrne K, et al. Afatinib versus gefitinib in patients with EGFR mutation-positive advanced non-small-cell lung cancer: overall survival data from the phase IIb LUX-Lung 7 trial. Ann Oncol 2017;28:270-7.

24. Schuler M, Wu YL, Hirsh V, et al. First-Line Afatinib versus Chemotherapy in Patients with Non-Small Cell Lung Cancer and Common Epidermal Growth Factor Receptor Gene Mutations and Brain Metastases. J Thorac Oncol 2016;11:380-90. 
25. Giovannetti E, Lemos C, Tekle C, et al. Molecular mechanisms underlying the synergistic interaction of erlotinib, an epidermal growth factor receptor tyrosine kinase inhibitor, with the multitargeted antifolate pemetrexed in non-small-cell lung cancer cells. Mol Pharmacol 2008;73:1290-300.

26. La Monica S, Madeddu D, Tiseo M, et al. Combination of Gefitinib and Pemetrexed Prevents the Acquisition of TKI Resistance in NSCLC Cell Lines Carrying EGFRActivating Mutation. J Thorac Oncol 2016;11:1051-63.

27. Li T, Ling YH, Goldman ID, Perez-Soler R. Scheduledependent cytotoxic synergism of pemetrexed and erlotinib in human non-small cell lung cancer cells. Clin Cancer Res 2007;13:3413-22.

28. Sirotnak FM, Zakowski MF, Miller VA, et al. Efficacy of cytotoxic agents against human tumor xenografts is markedly enhanced by coadministration of ZD1839 (Iressa), an inhibitor of EGFR tyrosine kinase. Clin Cancer Res 2000;6:4885-92.

29. Ciardiello F, Caputo R, Bianco R, et al. Antitumor effect and potentiation of cytotoxic drugs activity in human cancer cells by ZD-1839 (Iressa), an epidermal growth factor receptor-selective tyrosine kinase inhibitor. Clin Cancer Res 2000;6:2053-63.

30. Holleman MS, van Tinteren H, Groen HJ, et al. Firstline tyrosine kinase inhibitors in EGFR mutation-positive non-small-cell lung cancer: a network meta-analysis. Onco Targets Ther 2019;12:1413-21.

31. Reungwetwattana T, Nakagawa K, Cho BC, et al. CNS Response to Osimertinib Versus Standard Epidermal Growth Factor Receptor Tyrosine Kinase Inhibitors in Patients With Untreated EGFR-Mutated Advanced NonSmall-Cell Lung Cancer. J Clin Oncol 2018. [Epub ahead of print].

32. Akamatsu H, Koh Y, Ozawa Y, et al. Osimertinib With Ramucirumab in EGFR-mutated, T790M-positive Patients With Progression During EGFR-TKI Therapy: Phase Ib Study. Clin Lung Cancer 2018;19:e871-74.

33. Yu HA, Kim R, Makhnin A, et al. A phase 1/2 study of osimertinib and bevacizumab as initial treatment for patients with EGFR-mutant lung cancers. J Clin Oncol 2019;37:abstr 9086.

34. Neal JW, Hausrath D, Wakelee HA, et al. Osimertinib Plus Chemotherapy for EGFR-Mutant NSCLC at Progression:Safety Profile and Survival Analysis. J Clin Oncol 2019;37:abstr 9083.

35. Erickson AW, Brastianos PK, Das S. Assessment of
Effectiveness and Safety of Osimertinib for Patients With Intracranial Metastatic Disease: A Systematic Review and Meta-analysis. JAMA Netw Open 2020;3:e201617.

36. Mitsudomi T, Morita S, Yatabe Y, et al. Gefitinib versus cisplatin plus docetaxel in patients with non-small-cell lung cancer harbouring mutations of the epidermal growth factor receptor (WJTOG3405):an open label, randomised phase 3 trial. Lancet Oncol 2010;11:121-8.

37. Maemondo M, Inoue A, Kobayashi K, et al. Gefitinib or chemotherapy for non-small-cell lung cancer with mutated EGFR. N Engl J Med 2010;362:2380-8.

38. Zhou C, Wu YL, Chen G, et al. Erlotinib versus chemotherapy as first-line treatment for patients with advanced EGFR mutation-positive non-small-cell lung cancer (OPTIMAL, CTONG-0802): a multicentre, open-label, randomised, phase 3 study. Lancet Oncol 2011;12:735-42.

39. Rosell R, Carcereny E, Gervais R, et al. Erlotinib versus standard chemotherapy as first-line treatment for European patients with advanced EGFR mutation-positive non-small-cell lung cancer (EURTAC): a multicentre, open-label, randomised phase 3 trial. Lancet Oncol 2012;13:239-46.

40. Sequist LV, Yang JC, Yamamoto N, et al. Phase III study of afatinib or cisplatin plus pemetrexed in patients with metastatic lung adenocarcinoma with EGFR mutations. J Clin Oncol 2013;31:3327-34.

41. Wu YL, Zhou C, Hu CP, et al. Afatinib versus cisplatin plus gemcitabine for first-line treatment of Asian patients with advanced non-small-cell lung cancer harbouring EGFR mutations (LUX-Lung 6): an open-label, randomised phase 3 trial. Lancet Oncol 2014;15:213-22.

42. Shi YK, Wang L, Han BH, et al. First-line icotinib versus cisplatin/pemetrexed plus pemetrexed maintenance therapy for patients with advanced EGFR mutation-positive lung adenocarcinoma (CONVINCE): a phase 3, open-label, randomized study. Ann Oncol 2017;28:2443-50.

(English Language Editor: J. Reynolds)

Cite this article as: Dai L, Luo CY, Hu GX, Chen G, Wu CX, Yin J, Jiang ZY, Hu GF, Zhao J, Fu WF. Comparative analysis of first-line treatment regimens for advanced EGFRmutant non-small cell lung cancer patients with stable brain metastases. Ann Palliat Med 2020;9(4):2062-2071. doi: 10.21037/apm-20-1136 


\section{Supplementary}

Table S1 Literature search criteria

(((()“Carcinoma, Non-Small-Cell Lung”[Mesh]) OR (((()(((non-small-cell lung cancer[Title]) OR (non-small cell lung cancer[Title])) OR (non small-cell lung cancer[Title])) OR (non small cell lung cancer[Title])) OR (non-small-cell lung carcinoma[Title])) OR (non-small cell lung carcinoma[Title])) OR (non small-cell lung carcinoma[Title])) OR (non small cell lung carcinoma[Title])) OR (nsclc[Title]))) AND ((epidermal growth factor receptor[title/abstract]) OR (EGFR[Title/Abstract]))) AND (((()(((()((((treatment[Title/Abstract]) OR (therapy[Title/Abstract])) OR (tyrosine kinase inhibitor)) OR (TKI[Title/Abstract])) OR (osimertinib[Title/Abstract])) OR (dacomitinib[Title/Abstract])) OR (afatinib [Title/Abstract])) OR (erlotinib[Title/Abstract])) OR (gefitinib[Title/Abstract])) OR (icotinib[Title/Abstract])) OR (chemotherapy[Title/Abstract])) OR (first-line[Title/Abstract])) OR (first line[Title/Abstract])) OR (treatment-naive[Title/Abstract])) OR (treatment-naïve[Title/Abstract])) OR (untreated[Title/Abstract]))) AND (((((compare[Title/Abstract]) OR (comparison[Title/Abstract])) OR (comparative[Title/Abstract])) OR (comparing[Title/Abstract])) OR (versus[Title/Abstract])) OR (vs[Title/Abstract]))) AND ((((((Randomized Controlled Trial[Publication Type]) OR (controlled clinical trial[Publication Type])) OR (randomized[Title/Abstract])) OR (randomised[Title/Abstract])) OR (randomly [Title/Abstract])) OR (trial[Title/Abstract])) OR (phase[Title/Abstract])) AND (“0001/01/01”[Date - Publication] : “2019/12/22”[Date - Publication])

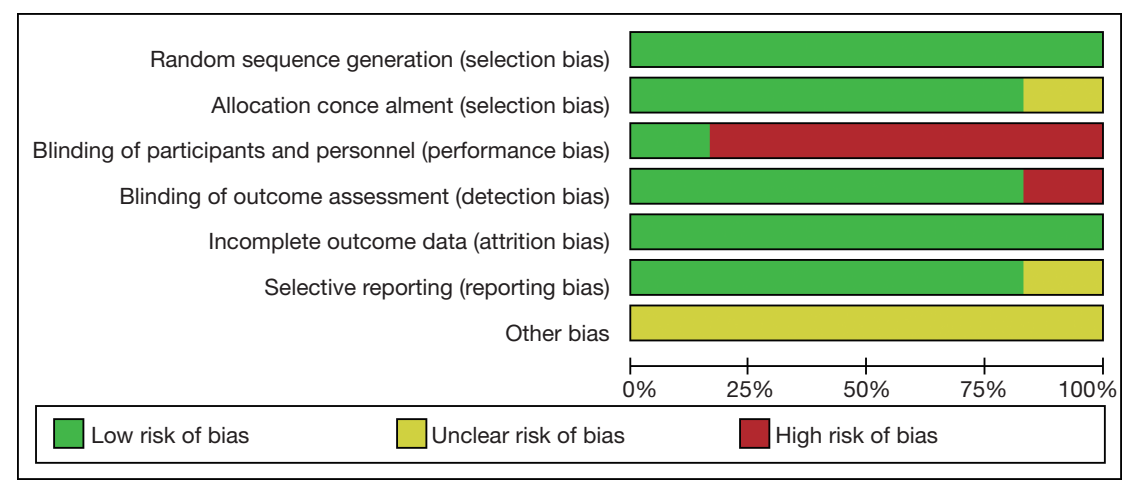

Figure S1 Cochrane risk of bias tool assessment.

Table S2 Quality and risk of bias assessment

\begin{tabular}{|c|c|c|c|c|c|c|c|}
\hline Study & $\begin{array}{l}\text { Sequence } \\
\text { generation }\end{array}$ & $\begin{array}{c}\text { Allocation } \\
\text { concealment }\end{array}$ & $\begin{array}{l}\text { Blinding of } \\
\text { participants }\end{array}$ & $\begin{array}{c}\text { Blinding of } \\
\text { outcome data }\end{array}$ & $\begin{array}{c}\text { Incomplete } \\
\text { outcome data }\end{array}$ & $\begin{array}{l}\text { Selective } \\
\text { reporting }\end{array}$ & Other sources of bias \\
\hline FLAURA (2019) & Low risk & Low risk & Low risk & Low risk & Low risk & Low risk & $\begin{array}{l}\text { Unclear risk (data from } \\
\text { subgroup analysis) }\end{array}$ \\
\hline NEJ026 (2019) & Low risk & Low risk & High risk & Low risk & Low risk & Low risk & $\begin{array}{l}\text { Unclear risk (data from } \\
\text { subgroup analysis) }\end{array}$ \\
\hline Lux-Lung 7 (2017) & Low risk & Low risk & High risk & Low risk & Low risk & Low risk & $\begin{array}{l}\text { Unclear risk (data from } \\
\text { subgroup analysis) }\end{array}$ \\
\hline Lux-Lung 6 (2016) & Low risk & Low risk & High risk & High risk & Low risk & Low risk & $\begin{array}{l}\text { Unclear risk (data from } \\
\text { subgroup analysis) }\end{array}$ \\
\hline
\end{tabular}


Table S3 Bayesian ranking results of network meta-analysis

\begin{tabular}{|c|c|c|c|c|c|c|c|}
\hline Treatment & \multicolumn{7}{|c|}{ Rank of possibility (\%) } \\
\hline \multicolumn{8}{|c|}{ Progression-free survival for stable brain metastases from advanced EGFR-mutated patients } \\
\hline Osimertinib & 22 & 37 & 17 & 10 & 7 & 4 & 3 \\
\hline S EGFR-TKI & 0 & 2 & 11 & 26 & 28 & 24 & 9 \\
\hline Gefitinib + PC & 57 & 22 & 9 & 6 & 3 & 2 & 1 \\
\hline Afatinib & 6 & 14 & 24 & 23 & 23 & 8 & 1 \\
\hline Gemcitabine + cis & 4 & 5 & 7 & 9 & 11 & 21 & 43 \\
\hline Pemetrexed + cis & 4 & 6 & 8 & 10 & 13 & 27 & 33 \\
\hline S EGFR-TKI & 1 & 11 & 29 & 24 & 20 & 14 & - \\
\hline Gefitinib + PC & 47 & 25 & 13 & 7 & 5 & 3 & - \\
\hline Afatinib & 1 & 6 & 13 & 23 & 34 & 22 & - \\
\hline Gemcitabine + cis & 14 & 15 & 14 & 17 & 17 & 24 & - \\
\hline Pemetrexed + cis & 18 & 14 & 12 & 15 & 14 & 27 & - \\
\hline
\end{tabular}

S EGFR-TKI, standard EGFR-TKI; bev, bevacizumab; PC, pemetrexed + carboplatin; cis, cisplatin. 